\title{
On the effect of external perturbation on amino acid salt bridge: a DFT study
}

\author{
BISWA JYOTI DUTTA, NABAJIT SARMAH and PRADIP KR BHATTACHARYYA* \\ Department of Chemistry, Arya Vidyapeeth College, Guwahati, Assam 781 016, India \\ E-mail: prdpbhatta@yahoo.com
}

MS received 30 January 2017; revised 12 March 2017; accepted 22 March 2017

\begin{abstract}
Effect of external perturbation (in terms of external electric field and solvents) on the stability of lysine-aspartic acid salt bridge was analyzed by density functional theory. Because of solvation, interaction energy in the aqueous phase is much lower as compared to gas phase. Interaction energy as well as stability (measured in terms of global hardness, HOMO energy and total electronic energy) are observed to be sensitive towards the strength and direction of the applied electric field. Gap between HOMO energy of the acids and salt bridge also points towards the feasibility of hydrogen bonding.
\end{abstract}

Keywords. Salt bridge; DFT; DFRT; Electric field; PCM.

\section{Introduction}

The stabilization of molecular conformations by the attractive interaction between oppositely charged ions (e.g., salt bridges) is of relevance in many areas of science. In particular, biological systems such as proteins, often contain salt bridges between ionic side chains that govern their structure and functions. ${ }^{1,2}$ Salt bridges can occur intra-molecularly, between a charged amino acid side chain and other groups within the protein; or intermolecularly, between the amino acid side chain and charged groups on a ligand or other biomolecules. ${ }^{3}$ Salt bridges are instrumental in determining binding specificity during protein-protein association and it contributes to the energetic as well. Moreover, a salt bridge compensates to the free energy requirement for binding. ${ }^{4-7}$ Lysine (lys) often forms salt bridge with aspartic acid (asp) and the salt bridge plays crucial role in the structure of the proteins. During salt bridge formation, hydrogen bonding between the $-\mathrm{NH}_{3}^{+}$(of lys) group and $-\mathrm{COO}^{-}$(of asp) contribute to the strength of the salt bridge.

In recent years, Density Functional Theory (DFT) has found enormous application in the interpretation of chemical reactivity. ${ }^{8-14}$ Density Functional Reactivity Theory (DFRT) can be used to estimate reactivity parameters. These parameters, called reactivity descriptors, defined within the framework of density functional

\footnotetext{
*For correspondence
}

theory are global hardness (also called chemical hardness), electrophilicity, chemical potential, local softness, Fukui functions, etc. ${ }^{15}$ These descriptors have been tested by several research groups and are found to be very useful in rationalizing the reactivity patterns in the molecular systems. ${ }^{16-20}$ Geerlings et al., and Chattaraj et al., have reviewed the theoretical basis for these descriptors and their applicability. ${ }^{21,22}$ Some of the recent developments and applications of these descriptors are highly impresive. ${ }^{23-27}$

The ionic environment in cellular environment imparts some sort of electric field which affects the reactivity of the biomolecules. In a cellular environment, the biomolecules may experience electric field and their reactivity pattern as well as stability may be altered. Moreover, when tissue is exposed to external electric field, it gets damaged. ${ }^{28}$ Effect of external electric field on the stability and reactivity of different molecular systems have been studied earlier. ${ }^{29-31}$ Effect of external electric field on chemical reactions is also familiar. ${ }^{32-35}$ The effect of electric field on the chemical reactivity has been carried out in a number of occassions. ${ }^{36-46}$ Chattaraj and co-workers have observed the effect of electric field on the global and local reactivity indices and confirmed that electric field considerably affects all the local reactivity indices and thereby the site selectivity of attack. ${ }^{37-39} \mathrm{Pal}$ and coworkers have studied the behaviour of these descriptors in presence of external electric field as well as in solvent media. ${ }^{39-42}$ Accordingly, introduction of electric field influences both physical and chemical properties 
of various molecular systems. In addition, a number of studies describe the effect of external electric field on biological molecules. ${ }^{43-46}$ Moreover, the aqueous environment within the cell is expected to contribute to the stabilized the salt bridge.

Herein we have made an attempt to gauge the stability of the lysine-aspartic acid salt bridge (lys-asp) in terms of interaction energy and reactivity descriptors in presence of external perturbation by external electric field and solvents by using DFT and DFRT. As the structure of proteins is extremely important in biology, understanding the stability of the salt bridge in biological environment (which is mainly the polar and charged ions that produce the electric field) is thus expected to make an insight on to the structure of the proteins and hence bears importance.

\section{Theoretical and computational details}

In DFT, global hardness $(\eta)$ is defined as the second derivative of energy with respect to the number of electrons respectively. ${ }^{47,48}$ Use of finite difference approximation and Koopmans' theorem ${ }^{49}$ lead to the working formulae for $\eta$ as:

$$
\eta=\frac{\mathrm{E}_{L U M O}-\mathrm{E}_{H O M O}}{2}
$$

where, $\mathrm{E}_{\text {НОмO }}$ is the energy of the HOMO (highest occupied molecular orbital) and $\mathrm{E}_{L U M O}$ is energy of the lowest unoccupied molecular orbital. The geometrical minima of the species are obtained using $6-31+G(d, p)$ basis set with Becke three parameter exchange and Lee, Yang and Parr correlation functional (B3LYP) ${ }^{50,51}$ and is confirmed by the absence of any negative frequency. After locating the minima, single point energy calculations have been carried out at different external electric field values applied along six directions (positive and negative directions of $x, y$ and $z$ axis; the sign + means that the field is applied along + direction of the axis and sign means that the field is applied along the - direction of the axis). It was shown that the strength of biological systems such as DNA base pairs can experience intense local electric fields close to the order of $\sim 10^{10} \mathrm{Vm}^{-1} .{ }^{32}$ Therefore, chosen strength of the external field ranges from 0.00 a.u. to 0.01 a.u. $\left[1\right.$ a.u. $\left.=51.4 \mathrm{~V} / \AA=51.4 \times 10^{10} \mathrm{Vm}^{-1}\right]$.

Strength of hydrogen bonds is expressed in terms of interaction energy $\left(\Delta \mathrm{E}_{\text {int }}\right)$ which is calculated using super molecular approach [for $\mathrm{A}+\mathrm{B} \rightarrow \mathrm{AB}, \Delta \mathrm{E}_{\text {int }}=\left(\mathrm{E}_{\mathbf{A B}}\right)-$ $\left(\mathrm{E}_{\mathrm{A}}+\mathrm{E}_{\mathrm{B}}\right)$, where, $\mathrm{E}$ is the total energy of the corresponding species]. When interaction energies are calculated, basis set superposition error (BSSE) is taken into account by using counterpoise $=\mathrm{N} \cdot{ }^{32,52}$ To observe the impact of solvent media, we repeated our observations in aqueous phase using PCM (Polarizable Continuum Model). ${ }^{53-55}$ All the calculations have been carried out using Gaussian09. ${ }^{56}$

\section{Results and Discussion}

Hydrogen bonding interaction is primarily electrostatic in nature and electrostatic potential in an outer surface of a molecule will therefore be well suited for predicting the nature of this kind of interaction. Figure 1 presents the isosurface diagram (with 0.001 a.u. density) of lys and asp which depicts a negative charge region around the $-\mathrm{COO}^{-}$group, acting as $\mathrm{H}$-acceptor and a positive charge on the $-\mathrm{NH}_{3}^{+}$group which facilitate hydrogen bond formation between the two moieties.

It is important to note that the $-\mathrm{COO}^{-}$group of asp accepts hydrogen from the $-\mathrm{NH}_{3}^{+}$group of lys. The $\mathrm{N}$ $\mathrm{H}$ bond length in isolated lys molecule is $1.03 \AA$ and in the salt bridge with asp this distance becomes $1.63 \AA$ and the O-H bond length is observed to be $1.04 \AA$, (Figure 2). Thus, a single linear hydrogen bond formation is observed with $\theta(\angle \mathrm{NHO})=174.75^{\circ}$.

\subsection{Variation of total electronic energy and interaction energy}

The time-independent nonrelativistic electronic Hamiltonian, under the Born-Oppenheimer approximation can be written as:

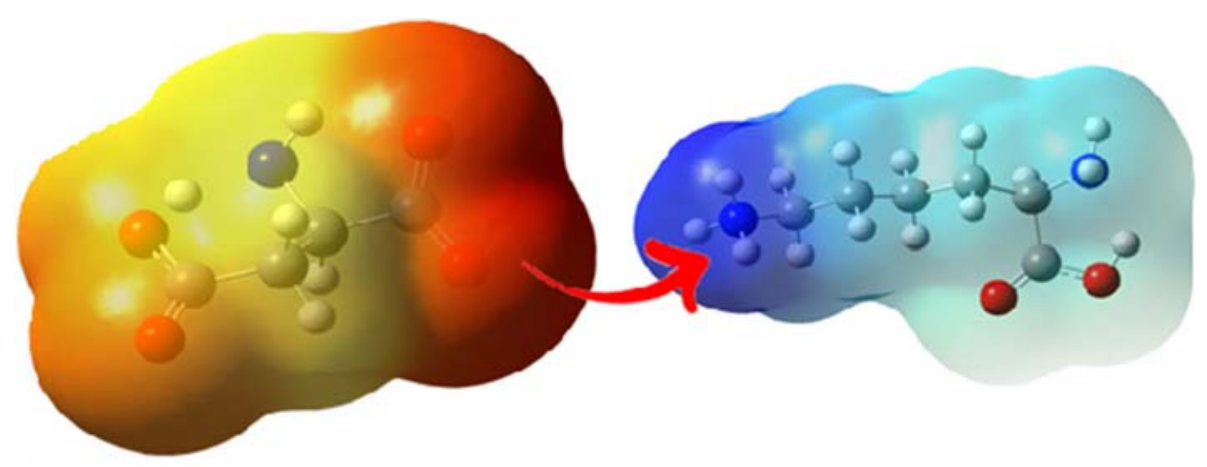

Figure 1. Isosurface diagram of lys and asp. 


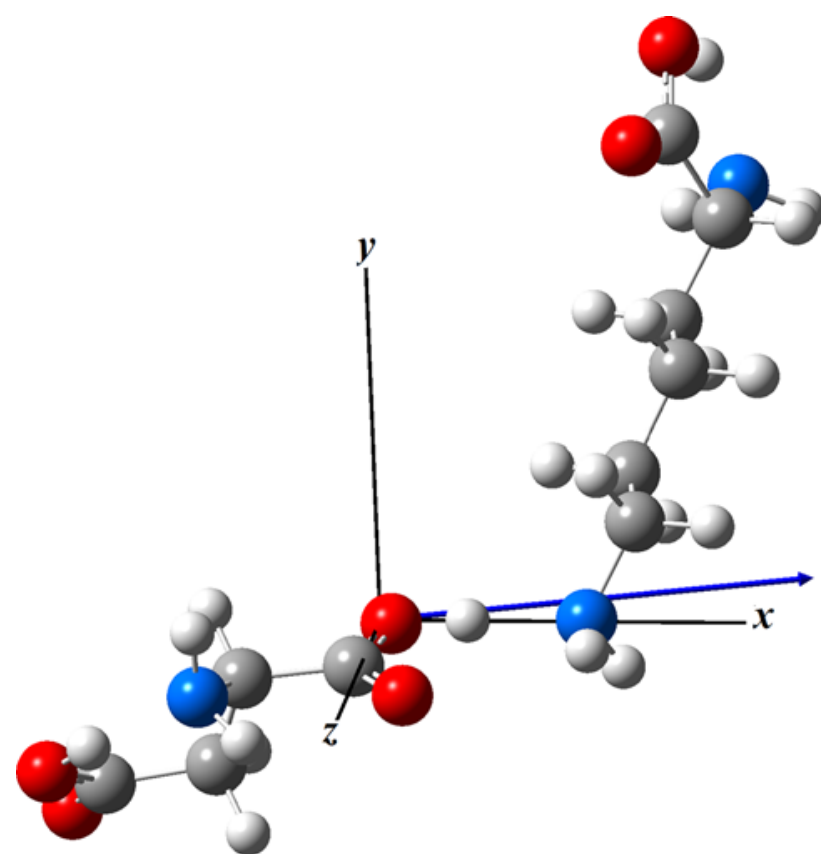

Figure 2. Gas phase optimised structure of the lys-asp salt bridge.

$$
\hat{H}=-\frac{1}{2} \sum_{i=1}^{N} \nabla_{i}^{2}-\sum_{i, A=1}^{N} \frac{Z_{A}}{r_{i A}}+\sum_{i<j}^{N} \frac{1}{r_{i j}}
$$

In the Hamiltonian, the external electric field is introduced as a perturbation parameter. The dipolar interaction of the Hamiltonian with the electric field, $F$ can be written as,

$$
\hat{H}=\hat{H}_{0}-\hat{d} \cdot \vec{F}
$$

where, $\hat{H}_{0}$ is the unperturbed Hamiltonian and $\hat{d}$ is the dipole moment operator. In the presence of external perturbation like external electric field, the total energy of the system can be written as,

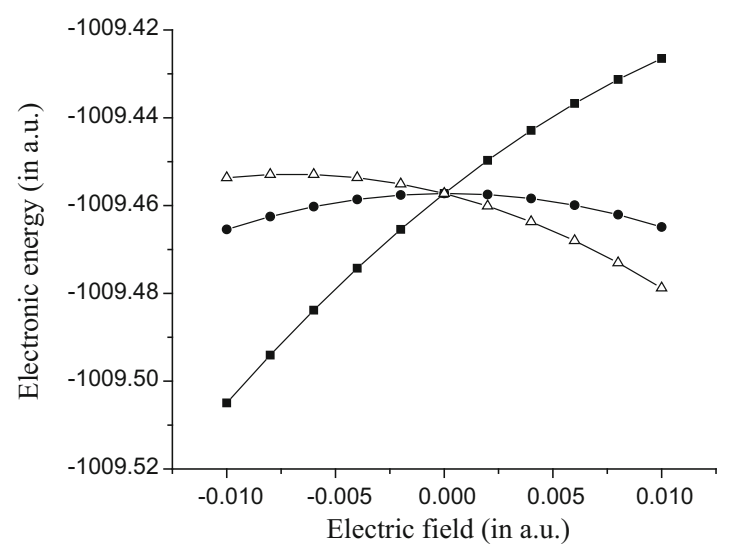

a

$$
E=E_{0}-\sum_{i} d_{i} F_{i}
$$

where, $E_{0}$ is the energy of the system at zero field, $d_{i}$ is the permanent dipole moment and $F_{i}$ is the strength of the field. The effect of the external electric field on total electronic energy can easily be estimated using equation (4). Total electronic energy $\left(\mathrm{E}_{\mathrm{el}}\right)$ of a system is a measure of stability of a system; accordingly, we studied the variation of total electronic energies of the salt bridge. It is interesting to note that application of external electric field on salt bridge in both gas and aqueous phases perturbs the $\mathrm{E}_{\mathrm{el}}$ of the system, (Figure 3).

Application of the electric field along $-x,-y$ and $+z$ directions stabilizes the salt bridge by lowering (more negative) the $\mathrm{E}_{\mathrm{el}}$ of the system in both the phases. A field of strength 0.010 a.u. applied along $-x$ direction drop the gas phase $\mathrm{E}_{\mathrm{el}}$ by 0.047748 a.u. $(29.96 \mathrm{kcal} / \mathrm{mol})$ and application of the same field strength along $-y$ and $+z$ directions results in drop of $\mathrm{E}_{\mathrm{el}}$ in gas phase by 0.008196 a.u. (5.14 kcal/mol) and 0.021563 a.u. (13.53 kcal $/ \mathrm{mol})$ respectively, (Figure 3a). The dipole moment of the salt bridge is calculated to be $10.5 \mathrm{D}$ and is aligned along the $x$-axis, Figure 2 . As can be realized from equation (4), that in presence of EEF, the $E_{e l}$ energy of a molecular system, depends on the dipole moment of the system. It is observed that the total electronic energy of the salt bridge is much sensitive towards the electric field applied along $x$-axis as compared to $y$-or $z$-axes. In essence, one must not ignore the effect of electric field while dealing with systems possessing high dipole moment. As the biomolecules possess substantial dipole moment and the presence of ions as well as the polar sugar-phosphate backbone and charged histones exert some electric field within the cellular environment, ${ }^{57}$ the obtained results confirm a significant role of electric

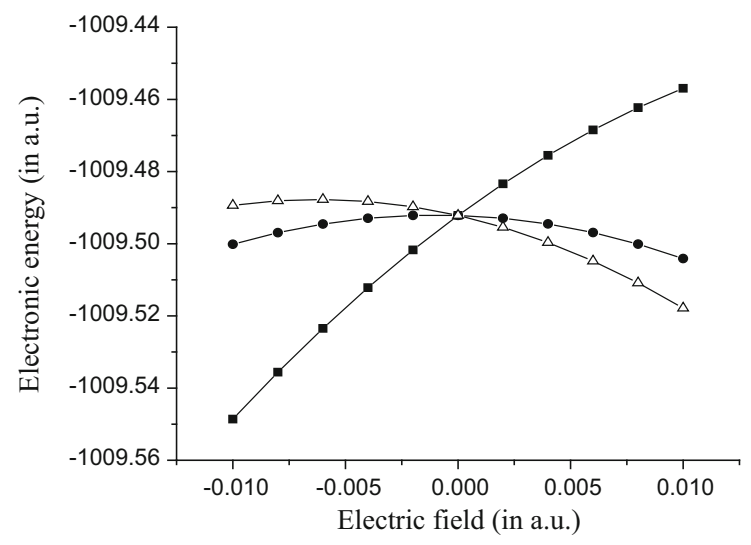

b

Figure 3. Variation of total electronic energy of the lys-asp salt bridge in, (a) gas and (b) aqueous phase with external electric field. ( $\square$-along $x$-axis, $\bullet$-along y-axis, $\Delta$-along $z$-axis). 
field on the energy of the molecular systems and hence on their stability.

Incorporation of aqueous phase does not play any significant role on the variation pattern of $\mathrm{E}_{\mathrm{el}}$, albeit, the aqueous phase energies are higher (more negative) than the gas phase values (Figure $3 a-3 b$ ). For example, a field of strength 0.01 a.u. applied along $-x,-y$ and $+z$ directions lowers the $\mathrm{E}_{\mathrm{el}}$ values by 0.0564 a.u. (35.43 $\mathrm{kcal} / \mathrm{mol}), 0.008208$ a.u. (5.15 kcal $/ \mathrm{mol})$ and 0.025705 a.u. (16.13 kcal $/ \mathrm{mol}$ ) respectively, (Figure $3 \mathrm{~b}$ ). Thus, the electronic energy of the system is observed to be sensitive towards the strength and orientation of the applied field and incorporation of aqueous phase stabilizes the salt bridge to some extent.

The gas phase interaction energies $\left(\Delta \mathrm{E}_{\text {int }}\right)$ are calculated to be too high; $-115.83 \mathrm{kcal} / \mathrm{mol}$. However, incorporation of aqueous phase results a spiky reduction in $\Delta \mathrm{E}_{\text {int }}$ (obtained from single point calculation in aqueous phase) owing to extensive solvation of the constituents and is calculated to be $-13.31 \mathrm{kcal} / \mathrm{mol}$. The considered amino acids bear charges $(+1$ charge in lys and -1 charge on asp) and the salt bridge bears no charge. An obvious impact of presence of charges on the amino acids is that the constituent amino acids undergo extensive solvation compared to the salt bridge which results a sharp fall in interaction energy. Usually, molecular systems involving charges or high dipole moment undergo extensive solvation in polar solvent media which leads to a sharp decrease in interaction energy. Earlier study also witnessed similar conclusion in case of interaction between aziridinium ion and nucleophiles. ${ }^{58}$ Further, to check the effect of aqueous medium on the proton transfer in the salt bridge, we re-optimized the geometry in the same solvent. The re-optimized geometry witnessed slight shifting of the proton to the $\mathrm{O}$ site. In gas phase, the $\mathrm{N} \cdots \mathrm{H}$ and $\mathrm{O} \cdots \mathrm{H}$ distances are 1.63 and $1.04 \AA$, respectively, which advocates transfer of the $\mathrm{NH}$ proton towards the $\mathrm{O}$ site in gas phase, and in the re-optimized structure in solvent phase these distances become 1.48 and $1.08 \AA$, respectively. Interaction energy calculated from the re-optimized geometry is $-12.24 \mathrm{kcal} / \mathrm{mol}$, not far apart from that obtained from single point calculation $(-13.31 \mathrm{kcal} / \mathrm{mol})$.

This result infers a hefty impact of a polar solvent on the interaction between the two components. Importantly, in both the phases (gas and aqueous) $\Delta \mathrm{E}_{\text {int }}$ of the salt bridge is well affected by the application of the electric field; pattern of variation of $\Delta \mathrm{E}_{\text {int }}$ with applied field strength in both gas and aqueous phase are similar (Figure 4).

In both the phases, maximum variation in $\Delta \mathrm{E}_{\text {int }}$ is observed when the field is applied along $x$ or $z$-axis. Interestingly, it is evident that the interaction energy sharply increases on changing the direction of applied field from $+x$ to $-x$ and from $-z$ to $+z$ direction. Application of the field along $y$ axis also leads to a variation in the interaction energy; however, variation is not as sharp as compared to the $x$ or $z$-axis. In aqueous phase, application of the field along $+x$ and $+z$ direction leads to a repulsive interaction and concomitant destabilization of the salt bridge. Thus, the direction of the applied field makes an important contribution towards $\Delta \mathrm{E}_{\mathrm{int}}$. In aqueous phase, application of the field (of strength 0.01 a.u.) along $-x,+y$ and $+z$ directions increase the $\Delta \mathrm{E}_{\text {int }}$ to -0.06735 a.u. $(-42.26 \mathrm{kcal} / \mathrm{mol})$, -0.02691 a.u. $(-16.88 \mathrm{kcal} / \mathrm{mol})$ and -0.05094 a.u. $(-31.96 \mathrm{kcal} / \mathrm{mol})$. The reverse is observed when the field is along $+x,-y$ and $-z$ directions; and at high field strength applied along $+x$ and $-z$ directions, a repulsive interaction is observed (positive $\Delta \mathrm{E}_{\text {int }}$ ), 0.026677 a.u. (16.74 kcal $/ \mathrm{mol})$ and 0.007676 a.u. $(4.81 \mathrm{kcal} / \mathrm{mol})$, respectively. Therefore, an inescapable role of solvent

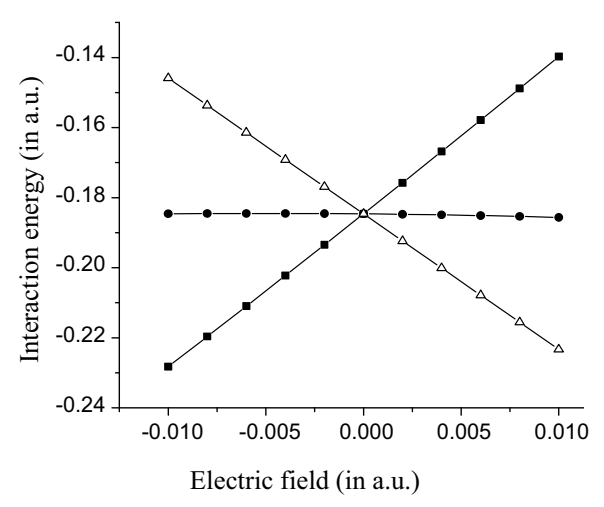

a

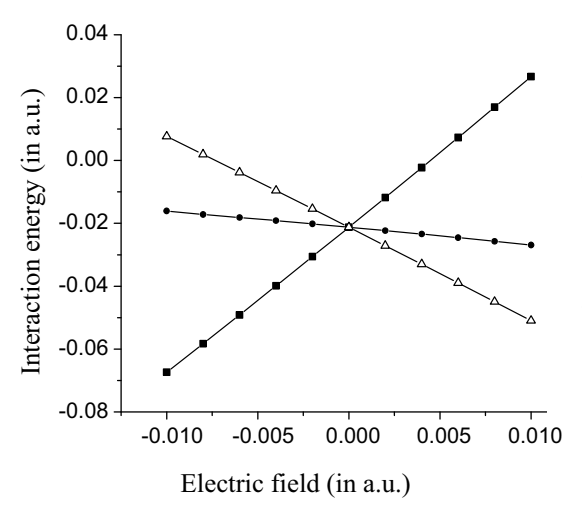

b

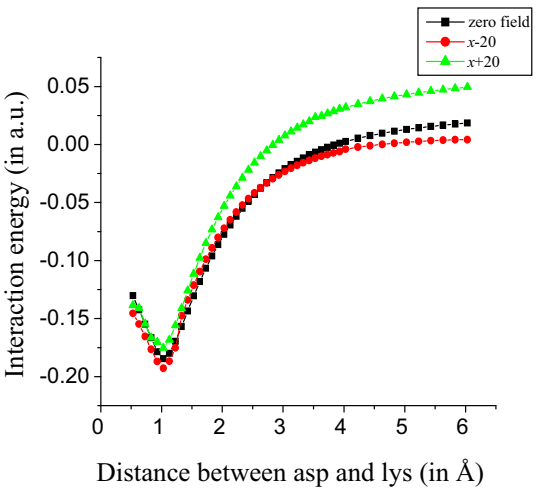

c

Figure 4. Variation of interaction energy in lys-asp in, (a) gas and (b) aqueous phase with external electric field, (c) variation of interaction energies with the separation distances between the two amino acid moieties. ( $\square$-along $x$-axis, $\bullet$-along $y$-axis, $\Delta$-along z-axis). 


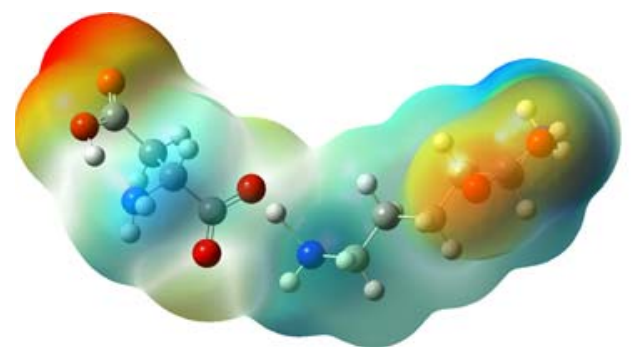

No field

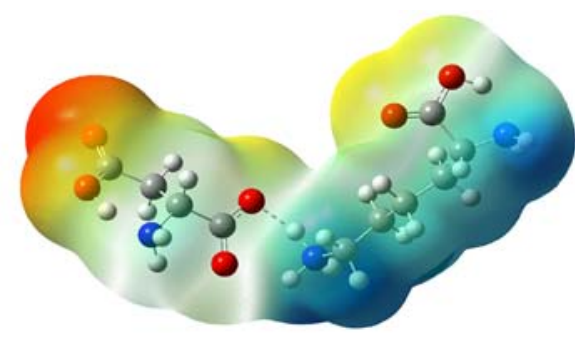

field $=x-0.010$

Figure 5. Change in the ESP surface in presence of electric field.

phase even in presence of external electric field can be construed from the calculated $\Delta \mathrm{E}_{\mathrm{int}}$ values.

Response of the $\Delta \mathrm{E}_{\text {int }}$ towards external electric field and solvent phase are different. A sharp drop in interaction energy is evident in presence of aqueous phase, whereas the impact of an external electric field on $\Delta E_{\text {int }}$ (rise/fall) depends on the orientation and strength of the applied field. This is on account of the directional nature of the electric field which is absent in case of the solvent. Results obtained advocate that the protein structure is affected in presence of external field as well as solvent phase. Further, we analyzed the variation of the interaction energy as a function of separation distances between the two amino acid moieties in gas phase and in presence of electric field of strength 0.0020 a.u. applied along $+x$ and $-x$ directions (Figure $4 c$ ). It is to be noted that as the two moieties approach each other, interaction energy increases and gets a maximum value at $1.04 \AA$.

The applied electric field interacts with the electron cloud and redistribution of the electron cloud may take place in presence of external electric field. This can easily be visualized with the help of ESP surface. A comparison of the ESP surfaces in absence of the electric field and at a field of strength 0.010 a.u. applied along $-x$ direction are shown in Figure 5. It can be seen from Figure 5 that electron cloud is redistributed in presence of the electric field and its impact on the interaction energy can be quantified using equation (4).

\subsection{Variation of reactivity parameters of the salt bridge}

Reactivity parameter of a system is indicative of their chemical stability and hence is important from chemical viewpoint. Variation of the global hardness of the salt bridge gas and aqueous phase with applied field strength along the three axes are presented in Figure $6 \mathrm{a}-\mathrm{b}$.

The gas phase global hardness of the salt bridge decreases noticeably on both sides of the axes. Upon application of external electric field along $x, y$ and $z$ axes, the global hardness decreases along both the direc- tions. But upon application of a field strength of 0.002 a.u. along $-x,-y$ and $+z$ directions, the global hardness increases slightly. Variation of the global hardness follows the same trend in both gas and aqueous phases, although aqueous phase hardness values are higher than those in the gas phase, indicating greater stability to the salt bridge in aqueous phase. For example, application of field strength of 0.01 a.u. along $-x$ direction raised the global hardness by 0.018485 a.u. $(11.59 \mathrm{kcal} / \mathrm{mol})$ in aqueous phase. According to the maximum hardness principle (MHP), maximum hardness leads to maximum stability. Here, in accordance with this principle, greater stability in the aqueous phase may be attributed to the dipole-dipole interaction between the salt bridge and solvent. MHP is found to be applicable in a wide range of molecular species. ${ }^{59-67}$ Earlier studies also confirmed that the MHP worked well in illustrating the chemical reactivity of drug molecules in ground state in presence of external electric field. ${ }^{68}$

This observation advocates that irrespective of increase/decrease of the total $\mathrm{E}_{\mathrm{el}}$, the overall stability of the salt bridges decreases on application of external electric field. However, unlike the variation of total $\mathrm{E}_{\mathrm{el}}$, reactivity parameter does not depend on the direction of the applied electric field but depends on the field strength only.

\subsection{HOMO-LUMO perspective}

To scrutinize the role of hydrogen bonding in the salt bridge formation, we have calculated the energy of the LUMO of lys (considering it as a H-donor); energy of HOMO of asp (considering it as a $\mathrm{H}$ acceptor). In the synergic effect of hydrogen bonding interactions of the type, $\mathrm{B}+\mathrm{HA} \leftrightarrow \mathrm{BHA}$ (where $\mathrm{B}$ is lys; HA is asp, BHA is the salt bridge), intermolecular hydrogen bond formation is favoured when the HOMO of the BHA has lower energy than the HOMO of B or LUMO of HA. Subsequently, the energy of separation, $\triangle \mathrm{E}\left(=\mathrm{E}_{\text {Номо }, \text { BнA }}-\mathrm{E}_{\text {НОмо, }}\right)$ in the absence of any external electric field, is calculated. Results are presented in 


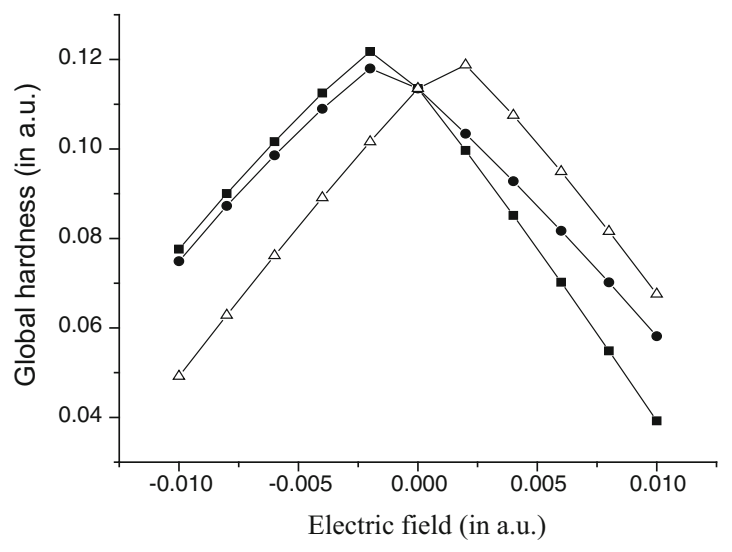

a

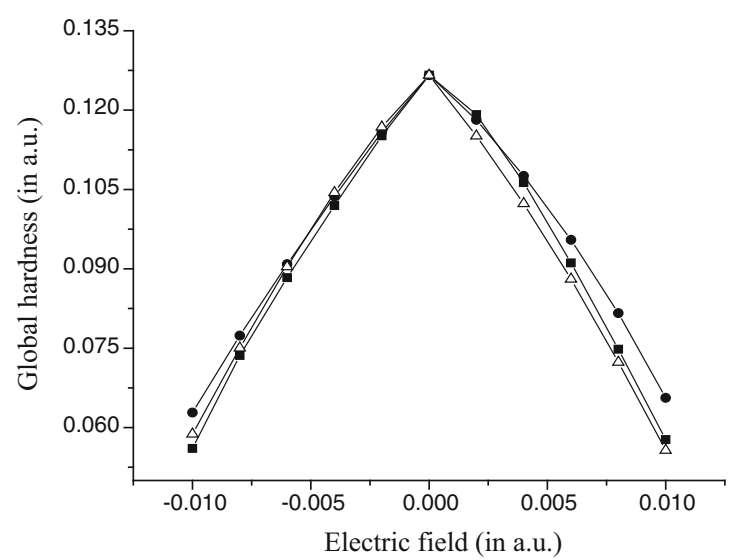

b

Figure 6. Variation of global hardness along three axes. (a) in gas phase; (b) in aqueous phase ( $\square$-along $x$-axis, $\bullet$-along y-axis, $\Delta$-along $z$-axis).

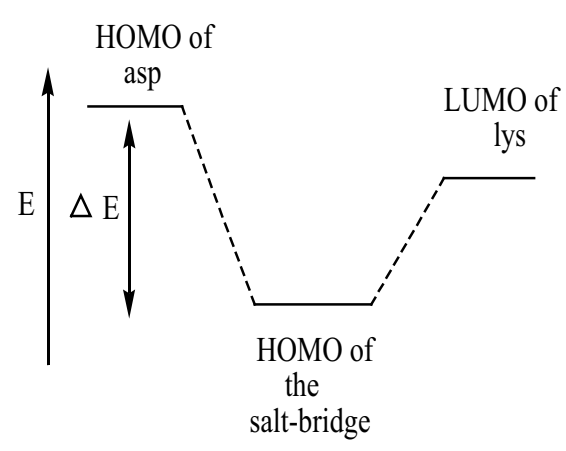

a

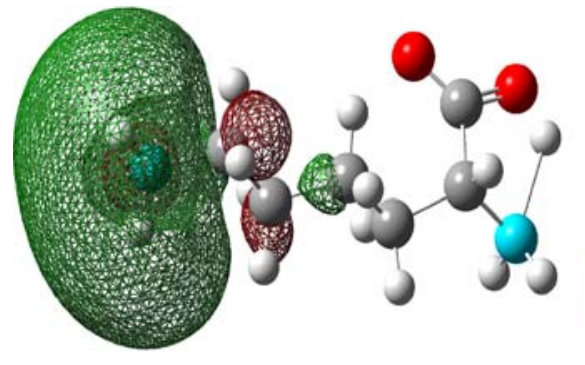

b

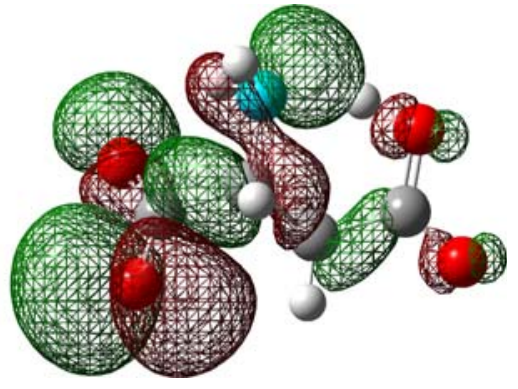

c

Figure 7. (a) Schematic presentation for energy separation of HOMO and LUMO during formation of salt-bridge; (b) LUMO of lys; (c) HOMO of asp.

Figure $7 \mathrm{a}$. The observed gas phase $\Delta \mathrm{E}$ values for asp is $-114.71 \mathrm{kcal} / \mathrm{mol}$ and in aqueous phase it is reduced to $-22.60 \mathrm{kcal} / \mathrm{mol}$. Earlier, Sarma et al. reported a correlation between $\Delta \mathrm{E}$ values and $\Delta \mathrm{E}_{\text {int }}$ (in case of hydrogen bonded system) which showed an increase in $\Delta \mathrm{E}_{\text {int }}$ with an increment in $\Delta \mathrm{E}$ values. ${ }^{69}$ Here, a large gas phase $\Delta \mathrm{E}$ valuecomplements to high magnitude of gas phase $\Delta \mathrm{E}_{\text {int }}$ (>-100 kcal/mol). Incorporation of aqueous phase lowers $\Delta \mathrm{E}$ values $(\sim-20 \mathrm{kcal} / \mathrm{mol})$, thus supporting the observed lowering in $\Delta \mathrm{E}_{\text {int }}(-13.33 \mathrm{kcal} / \mathrm{mol})$. Sharp drop in $\Delta \mathrm{E}$ is due to the fact that the salt bridge possesses large dipole moment (10.5 D) and strong dipole-dipole interaction between solvent and the salt bridge stabilizes the $\mathrm{E}_{\text {номо }}$ of the salt bridge and results in much lower $\Delta \mathrm{E}$ value in aqueous phase compared to the gas phase value. However, no linear relationship between $\Delta \mathrm{E}$ and $\Delta \mathrm{E}_{\text {int }}$ is observed. Apart from $\Delta \mathrm{E}$ values, it is worthwhile to study the shape of the LUMO of the donor and HOMO of the acceptor. Figure $7 b-c$ reveal that LUMO of lys is localized over the $-\mathrm{NH}_{3}^{+}$group, and this facilitates the $-\mathrm{NH}_{3}^{+}$group to participate in hydro- gen bonding as a hydrogen donor. Moreover, HOMO of asp is spreading over $-\mathrm{COO}^{-}$group (Figure 7c), which makes them hydrogen acceptor during hydrogen bonding formation. Importantly, the HOMO of the salt bridge is lying far below the LUMO of lys, favouring hydrogen bond formation from the HOMO-LUMO energy perspectives.

It is expected that the HOMO of the salt bridge and asp are affected by the presence of electric field. Figure 8 shows the variation of HOMO energy of asp and the corresponding lys-asp salt bridge. It is also seen that the HOMO of the salt bridge is well affected by the presence of electric field. In both gas and aqueous phases, we observe destabilization of the HOMO (Figure 8a,b). In contrast to the salt bridge, variation of HOMO of asp follows a different pattern; drop in HOMO energy is observed upon application of the field along $-x$ and $-z$ directions in asp. Interestingly, presence of aqueous phase does not change the variation pattern (Figure 8c,d). However, a drop in $\mathrm{E}_{\text {номо }}$ and therefore, a more stable $\mathrm{HOMO}$ results in aqueous 


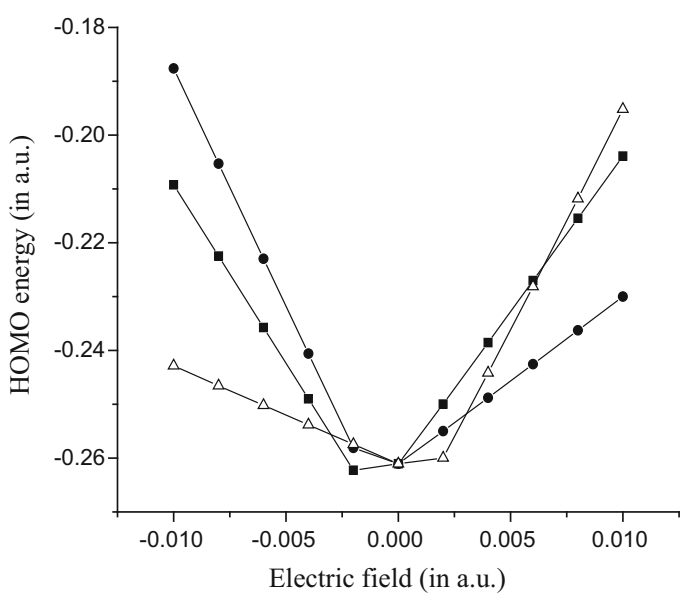

a

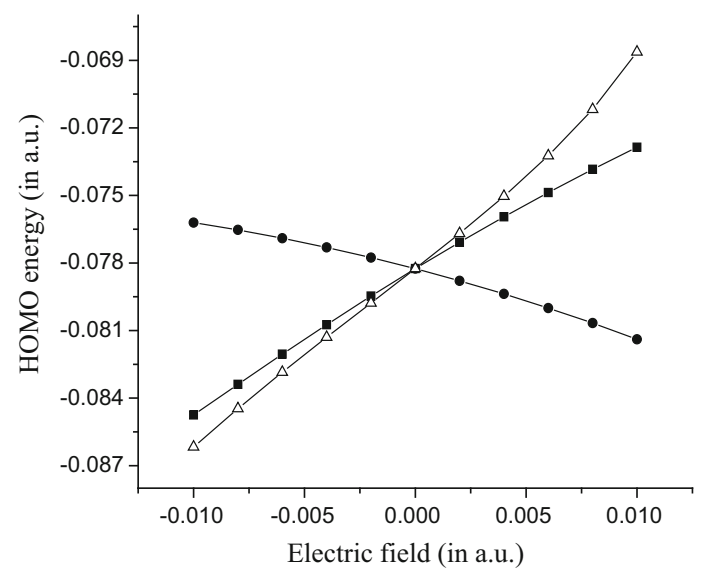

c

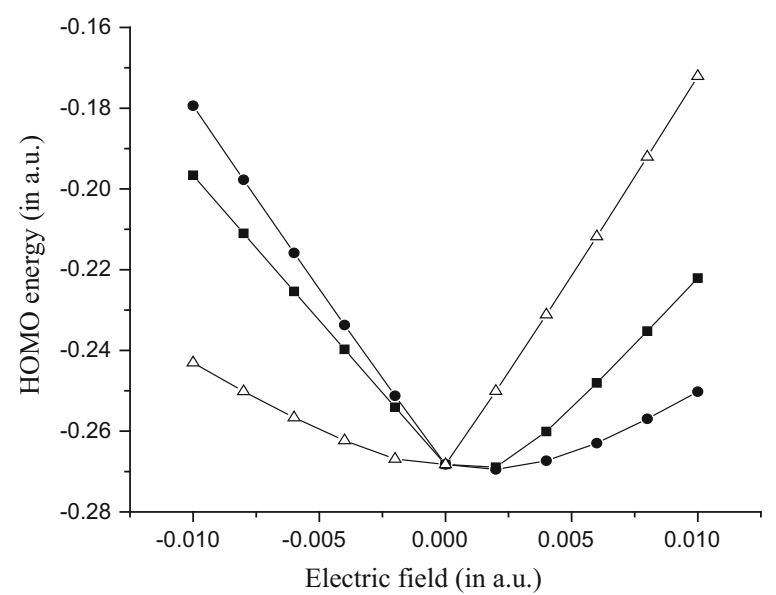

b

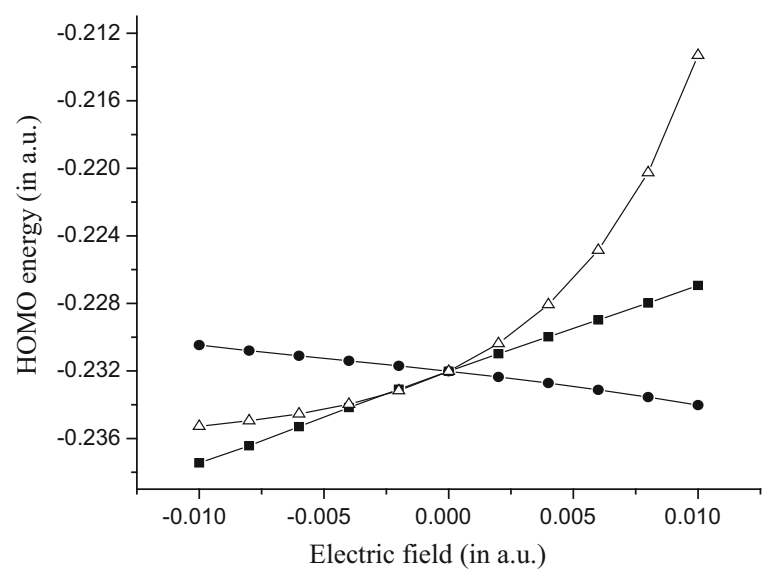

d

Figure 8. Variation of HOMO energy with external electric field. (a) lys-asp salt bridge in gas phase; (b) lys-asp salt bridge in aqueous phase; (c) asp in gas phase; and (d) asp in aqueous phase. ( $\boldsymbol{\square}$-along $x$-axis, $\bullet$-along y-axis, $\Delta$-along z-axis).

phase. This variation explains why $\Delta \mathrm{E}$ value (Figure $7 \mathrm{a}$ ) is perturbed in the presence of external electric field which in turn affects the strength of the hydrogen bonding in the salt bridge.

\section{Conclusions}

Our study found out some important aspects on the stability of amino acid salt bridge of lys and asp upon application of external electric field and in aqueous phase. Calculated interaction energies confirm formation of salt bridge in the gas phase. Incorporation of aqueous phase reduces the interaction energies considerably. Total electronic energy and interaction energies are observed to be sensitive towards the strength and direction of the applied electric field; however, presence of aqueous phase does not alter the variation pattern. Variation of global hardness suggests that application of external electric field imparts instability to the salt bridge. The study also delineates the inherent feasibility of formation of hydrogen bonding during formation of salt bridge. Generic conclusion that can be drawn from this study is that aqueous phase and external electric field essentially contribute to the stability of the salt bridge and hence it is expected that the structure and the stability of the protein are also affected by the presence of such external perturbation.

\section{Acknowledgements}

Authors thank the Department of Science and Technology (DST), India for the financial grant (No. SB/S1/PC-17/2014).

\section{References}

1. Kumar S and Nussinov R 1999 Salt bridge stability in monomeric proteins J. Mol. Biol. 931241 
2. Christie J M, Arvai A S, Baxter K J, Heilmann M, Pratt A J, O-Hara A, Kelly S M, Hothorn M, Smith B O, Hitomi K, Jenkins G I and Getzo E D 2012 Plant UVR8 photoreceptor senses UV-B by tryptophan-mediated disruption of cross-dimer salt bridges Science 3351492

3. Robert A 2000 In Copeland enzymes: A practical introduction to structure, mechanism, and data analysis (New York: Wiley-VCH)

4. Fersht A R 1984 Basis of biological specificity Biochem. Sci. 9145

5. Honig B and Yang A S 1995 Free energy balance in protein folding Adv. Protein Chem. $\mathbf{4 6} 27$

6. Gao J, Mammen M and Whitesides G M 1996 Evaluating electrostatic contributions to binding with the use of protein charge ladders Science $\mathbf{2 7 2} 535$

7. Xu D, Lin S L and Nussinov R 1997 Protein binding versus protein folding: The role of hydrophilic bridges in protein associations J. Mol. Biol. 26568

8. Kohn W, Becke A D and Parr R G 1996 Density functional theory of electronic structure J. Phys. Chem. 100 12974

9. Baerends E J and Gritsenko O V 1997 A quantum chemical view of density functional theory J. Phys. Chem. A 1015383

10. Chermette H 1998 Density functional theory: A powerful tool for theoretical studies in coordination chemistry Coord. Chem. Rev. 178699

11. Andrews L and Citra A 2002 Infrared spectra and density functional theory calculations on transition metal nitrosyls. Vibrational frequencies of unsaturated transition metal nitrosyls Chem. Rev. 102885

12. Ziegler T and Autschbach J 2005 Theoretical methods of potential use for studies of inorganic reaction mechanisms Chem. Rev. 1052695

13. Neese F 2009 Prediction of molecular properties and molecular spectroscopy with density functional theory: from fundamental theory to exchange-coupling Coord. Chem. Rev. 253526

14. Schultz N E, Zhao Y and Truhlar D G 2005 Density functionals for inorganometallic and organometallic chemistry J. Phys. Chem. A 10911127

15. Parr R G and Yang W 1989 Density functional theory of atoms and molecules (New York: Oxford University Press)

16. Chandra A K and Nguyen M T 2007 Use of DFT-based reactivity descriptors for rationalizing radical addition reactions: Applicability and difficulties Faraday Discuss. 135191

17. Molteni G and Ponti A 2003 Arylazide Cycloaddition to Methyl Propiolate: DFT-Based Quantitative Prediction of Regioselectivity Chem. Eur. J. 92770

18. Roy R K 2003 Nucleophilic substitution reaction of alkyl halides: a case study on density functional theory (DFT) based local reactivity descriptors J. Phys. Chem. A $\mathbf{1 0 7}$ 397

19. Nguyen H M T, Peeters J, Nguyen M T and Chandra A K 2004 Use of DFT-based reactivity descriptors for rationalizing radical reactions: A critical analysis J. Phys. Chem. A $\mathbf{1 0 8} 484$

20. Melin J, Aparicio F, Subramanian V, Galvan M and Chattaraj P K 2004 Is the Fukui Function a Right Descriptor of Hard- Hard Interactions? J. Phys. Chem. A 1082487
21. Geerlings P, Proft F D and Langenaekar W 2003 Conceptual density functional theory Chem. Rev. 1031793

22. Chattaraj P K, Sarkar U and Roy D R 2006 Electrophilicity index Chem. Rev. 1062065

23. Roy R K and Saha S 2010 Studies of regioselectivity of large molecular systems using DFT based reactivity descriptors Annu. Rep. Prog. Chem., Sect. C: Phys. Chem. 106118

24. Vijayaraj R, Subramanian V and Chattaraj P K 2009 Comparison of global reactivity descriptors calculated using various density functionals: A QSAR perspective J. Chem. Theor. Comput. 52744

25. Putz M V, Mingos D and Michael P 2012 (Eds.) Applications of density functional theory to chemical reactivity (Berlin: Springer)

26. Cohen M H and Wasserman A 2007 On the foundations of chemical reactivity theory J. Phys. Chem. A 111 2229

27. Ramya K R and Venkatnathan A 2012 Stability and reactivity of methane clathrate hydrates: insights from density functional theory J. Phys. Chem. A 1167742

28. Ross W 1993 Biological effects of electromagnetic fields J. Cell. Biochem. $\mathbf{5 1} 410$

29. Neog B, Sarmah N and Bhattacharyya P K 2014 Effect of external electric field on drug-guanine adduct: A conceptual density functional theory study J. Ind. Chem. Soc. 9195

30. Mazurkiewicz J and Tomasik P 2012 Effect of external electric field upon charge distribution, energy and dipole moment of selected monosaccharide molecules Natural Sci. 4276

31. Calvo F and Dugourd P 2008 Folding of gas-phase polyalanines in a static electric field: Alignment, deformations, and polarization effects Biophys. J. 118

32. Kinosita K J and Tsong T Y 1977 Hemolysis of human erythrocytes by transient electric field Proc. Natl. Acad. Sci. USA 741923

33. Alemani M, Peters M V, Hecht S, Rieder K H, Moresco F and Grill L 2006 Electric field-induced isomerization of azobenzene by STM J. Am. Chem. Soc. 12814446

34. Adey W R 1993 Biological effects of electromagnetic fields J. Cell. Biochem. 51410

35. Charles P and Elliots P 1995 In Handbook of biological effects of electromagnetic fields $2^{\text {nd }}$ Ed. (USA: CRC press)

36. Parthasarathi R, Subramanian V and Chattaraj P K 2003 Effect of electric field on the global and local reactivity indices Chem. Phys. Lett. 38248

37. Kramer K H and Bernstein R B 1964 Sudden Approximation Applied to Rotational Excitation of Molecules by Atoms. I. Low-Angle Scattering J. Chem. Phys. 40 200

38. Brooks P R and Jones M E 1966 Reactive scattering of $\mathrm{K}$ atoms from oriented CH3I molecules J. Chem. Phys. 453449

39. Kar R, Chandrakumar K R S and Pal S 2007 The Influence of electric field on the global and local reactivity descriptors: reactivity and stability of weakly bonded complexes J. Phys. Chem. A 111375

40. Kar R and Pal S 2008 Electric field response of molecular reactivity descriptors: A case study Theor. Chem. Acc. 120375 
41. Kar R and Pal S 2010 Effect of solvents having different dielectric constants on reactivity: A conceptual DFT approach Int. J. Quant. Chem. 1101642

42. Kar R and Pal S 2008 In External Field and Chemical Reactivity P K Chattaraj (Ed.) (Boca Raton: Taylor and Francis, CRC Press)

43. Ceróon-Carrasco J P, Cerezo J and Jacquemin D 2014 How DNA is damaged by external electric fields: selective mutation vs. random degradation Phys. Chem. Chem. Phys. 168243

44. Jissy A K and Datta A 2010 Designing molecular switches based on DNA-base mispairing J. Phys. Chem. B 11415311

45. Cerón-Carrasco J P and Jacquemin D 2013 Electric-field induced mutation of DNA: A theoretical investigation of the GC base pair Phys. Chem. Chem. Phys. 154548

46. Kanvah S, Joseph J, Schuster G B, Barnett R N, Cleveland C L and Landman U 2010 Oxidation of DNA: Damage to nucleobases Acc. Chem. Res. 43280

47. Parr R G and Pearson R G 1983 Absolute hardness: Companion parameter to absolute electronegativity J. Am. Chem. Soc. 1057512

48. Parr R G, Donnelly R A, Levy M and Palke W E 1978 Electronegativity: The density functional viewpoint $J$. Chem. Phys. 683801

49. Koopmans T 1934 Über die Zuordnung von Wellenfunktionen und Eigenwerten zu den einzelnen Elektronen eines Atoms Physica 1104

50. Becke A D 1993 Density-functional thermochemistry. III. The role of exact exchange J. Chem. Phys. 985648

51. Lee C, Yang W and Parr R G 1988 Development of the Colle-Salvetti correlation-energy formula into a functional of the electron density Phys. Rev. B 37785

52. Galano A and Alvarez-Idaboy J R 2006 A new approach to counterpoise correction to BSSE J. Comput. Chem. 27 1203

53. Miertus S, Scrocco E and Tomasi J 1981 Electrostatic interaction of a solute with a continuum. A direct utilizaion of $\mathrm{AB}$ initio molecular potentials for the prevision of solvent effects J. Chem. Phys. 55117

54. Mennucci B and Tomasi J 1997 Continuum solvation models: A new approach to the problem of solute's charge distribution and cavity boundaries J. Chem. Phys. 1065151

55. Cammi R, Mennucci B and Tomasi J 2000 Fast evaluation of geometries and properties of excited molecules in solution: A Tamm-Dancoff model with application to 4-dimethylaminobenzonitrile J. Phys. Chem. A 1045631

56. Frisch M J et al. 2010 Gaussian 09, Revision B.01, Gaussian, Inc., Wallingford, CT.

57. Arabi A A and Matta C F 2011 Effects of external electric fields on double proton transfer kinetics in the formic acid dimer Phys. Chem. Chem. Phys. 1313738

58. Sarmah N, Neog B and Bhattacharyya P K 2011 Affinity of aziridinium ion towards different nucleophiles: A density functional study Comput. Theor. Chem. 97630

59. Parr R G and Chattaraj P K 1991 Principle of maximum hardness J. Am. Chem. Soc. 1131854

60. Chamorro E, Chattaraj P K and Fuentealba P 2003 Variation of the electrophilicity index along the reaction path J. Phys. Chem. A $\mathbf{1 0 7} 7068$

61. Sinha S and Bhattacharyya P K 2014 Alkylation of guanine by formononetin nitrogen mustard derivatives: A DFT study Comput. Theor. Chem. 1027135

62. Parthasarathi R, Padmanabhan J, Subramanian V, Maiti B and Chattaraj P K 2003 Chemical reactivity profiles of two selected polychlorinated biphenyls J. Phys. Chem. A 10710346

63. Bhattacharyya P K 2015 Reactivity, aromaticity and absorption spectra of pillar [5] arene conformers: A DFT study Comput. Theor. Chem. 106620

64. Ash S, Beg H, Mazumdar P, Salgado-Morán G and Misra A 2014 Polarizability, hardness and electrophilicity as global descriptors for intramolecular proton transfer reaction path Comput. Theor. Chem. 103150

65. Chattaraj P K and Poddar A 1998 A density functional treatment of chemical reactivity and the associated electronic structure principles in the excited electronic states J. Phys. Chem. A $\mathbf{1 0 2} 9944$

66. Bhattacharyya P K and Kar R 2011 Does structural variation in the aziridinium ion facilitate alkylation? Comput. Theor. Chem. 9675

67. Jayakumar N and Kolandaivel P 2000 Studies of isomer stability using the maximum hardness principle (MHP) Int. J. Quant. Chem. 76648

68. Neog B, Sarmah N, Kar R and Bhattacharyya P K 2011 Effect of external electric field on aziridinium ion intermediate: A DFT study Comput. Theor. Chem. 96760

69. Sarma R, Bhattacharyya P K and Baruah J B 2011 Short range interactions in molecular complexes of 1 , 4-benzenediboronic acid with aromatic N-oxides Comput. Theor. Chem. 963141 